\title{
Research on Tacit Knowledge Sharing of Enterprise R\&D Team under Network Environment
}

\author{
Jianhao $\mathrm{Li}^{\mathrm{a})}$ \\ College of Management, Chongqing University of Technology, Chongqing 400054, China \\ a) Corresponding author: 364927471@qq.com
}

\begin{abstract}
For enterprises, restricted by various factors, tacit knowledge cannot be effectively explicit and utilized in enterprises, which is a great waste of knowledge. Therefore, extracting the characteristics of tacit knowledge and constructing corresponding enterprise tacit knowledge database can facilitate the sharing, communication and improvement among employees in the enterprise. In order to extract the tacit knowledge, this paper proposes a TFIDF recessive knowledge feature extraction method based on Euclidean distance calculation. After extracting the features from the traditional TFIDF method, the relationship between different features is taken into consideration, and the weight value is further optimized by Euclidean distance. After extracting the characteristics of tacit knowledge, this paper puts forward the method of constructing the tacit knowledge sharing system in the enterprise R \& D Department of the pattern recognition. Through the pattern recognition method, it is convenient to improve the enterprise tacit knowledge database, which is of great significance for the enterprise employees to communicate with each other.
\end{abstract}

Key words: Tacit Knowledge; Euclidean Distance; TFIDF; Pattern Recognition.

\section{INTRODUCTION}

Tacit knowledge exists in all sectors, and the proportion of tacit knowledge and explicit knowledge is as high as 9:1[1]. Nowadays, with the rapid development of Internet, how to excavate and share tacit knowledge inside enterprises has become an urgent problem [2]. By tacit knowledge explicit and sharing within the enterprise, it can effectively enhance the soft cultural strength of enterprises. On the basis of the introduction of tacit knowledge, this paper puts forward the method of extracting TFIDF tacit knowledge feature based on Euclidean distance calculation and puts forward the construction method of the tacit knowledge sharing system in the enterprise R \& D Department of the pattern recognition, which is of great significance to the knowledge sharing of the enterprise.

\section{RESEARCH ON TACIT KNOWLEDGE IN NETWORK ENVIRONMENT}

With the development of Internet technology, there are more and more channels for enterprises and users to acquire knowledge, and it is more and more convenient to acquire knowledge. For enterprises, explicit knowledge is more easily obtained, tacit knowledge is limited by its characteristics and cannot be effectively tapped, but the value of tacit knowledge cannot be ignored [3].

\section{Knowledge Sharing in Network Environment}

Just 20 years, the Internet has crossed from the initial era of Web1.0 to Web3.0 era and is about to enter the Web4.0 era [4]. Just 20 years, the Internet has crossed from the initial era of Web1.0 to Web3.0 era and is about to enter the Web4.0 era. The current society is an information society that shines brilliantly on the Internet. With the support of artificial intelligence, various types of equipment are connected to each other, and a "person-network- 
device" interconnected intelligent network has been formed. People-to-people communication, People-to-equipment has become easier and more accurate.

There are many different understandings of the term knowledge sharing. In general, people regard knowledge sharing as a process of mutual exchange or mutual transformation. Taking the enterprise as the research object, Bartol believes that employees are the core and source of knowledge sharing. Knowledge sharing is the exchange of knowledge and skills among employees. Hooff believes that knowledge sharing is an explicit tacit knowledge that employees exchange with each other. Based on the process of further innovation, Ipe believes that knowledge sharing is the process of transforming employees' own knowledge into knowledge of other employees [5] [6] [7].

Under the contemporary network environment, the concept of "people-oriented", "everyone can create" and "everyone participates in sharing" is reflected in the concept of socialized cooperation and the promotion of internal and external comprehensive communication and dialogue. The Web3.0 era pays attention to build users to build their own personality network according to their own habits and hobbies, which facilitates users to obtain the knowledge they need, and thus greatly facilitates the re-creation of knowledge. In the past, the dissemination of knowledge relied mainly on books, televisions, and face-to-face experience exchanges. However, the development of the Internet made it possible for people and people to communicate without regard to distance. For example, the rise of MOOCs [8] and various open class platforms allows people to learn at any time as long as they have a network. Chat software such as WeChat allows people to communicate texts, voices, images, and videos anytime, anywhere. To the extent that it brings convenience to people's knowledge acquisition and exchange.

\section{The Connotation and Value of Tacit Knowledge}

In 1958, Michael Polanyi first put forward the concept of "tacit knowledge" and divided human knowledge into two categories: tacit knowledge and explicit knowledge. Polanyi believes that tacit knowledge belongs to the individual itself, which is related to the life learning experience and environment of the individual. It is not as logical as explicit knowledge, and it is difficult to share its expression and communication. People can share some tacit knowledge into explicit knowledge through thinking and sorting, but compared with tacit knowledge, explicit knowledge is very poor. This is why people think that "know much more than they say", Drucker and Dulke believe that although implicit knowledge exists, it cannot be shown as clearly as explicit knowledge. Even repeated practice can only comprehend part of the tacit knowledge, and the sharing of tacit knowledge will be shared by the sharing. The restrictions on the ability to enjoy will also be affected by the knowledge recipient's ability to comprehend [9].

\section{CONSTRUCTION OF TACIT KNOWLEDGE SHARING SYSTEM OF ENTERPRISE R\&D TEAM}

With the rapid development of the Internet, the scale of enterprises is increasing. Although the internal communication methods of enterprises are also increasing, there are inevitably obstacles in the communication between various departments, and there are difficulties in the exchange of knowledge. Especially for R\&D departments of enterprises, if it is impossible to effectively use the tacit knowledge of R\&D teams, it is a great waste of knowledge. Therefore, it is of great significance to build a tacit knowledge sharing system for corporate R\&D teams.

\section{Tacit Knowledge Feature Extraction}

To build a tacit knowledge sharing system, we must first consider how to effectively extract tacit knowledge. The extraction of traditional tacit knowledge mainly depends on people's experience, and there is no stable method and system for feature extraction [10] [11].

In order to mine the knowledge of tacit knowledge, the knowledge feature extraction based on ontology structure is adopted. Ontology structural characteristics can be expressed as

$$
\left\{C, R, H^{c}, \operatorname{Rel}, A^{\circ}\right\}
$$


Where $C$ represents a concept, $R$ represents the relationship between concepts, $H^{c}$ is the classification relationship between concepts, $R e l$ for its non-categorical relationship, $A^{o}$ is an axiom that defines the rules for interpreting different relationships.

For different fields, the corresponding tacit knowledge systems are often different. Therefore, it is necessary to formulate relevant tacit knowledge classification according to different research fields and analyze the relationship among each category, including dependence, association, aggregation, combination, inheritance, and achieving six kinds of relationships. The feature extraction of different tacit knowledge includes TFIDF method, word frequency method, information gain method, and chi-square statistic method. This paper adopts the TFIDF feature extraction method based on the Euclidean distance. The research and development team under the network environment is used as the research background. The classification of the recessive knowledge in the programming language can be divided into: $\mathrm{C}++$ language class, Python language class, $\mathrm{C}$ language class, etc. The feature extraction is performed on the acquired knowledge using the TFIDF method [12]. If a certain feature is extracted as $F_{1}$, the frequency of occurrence of the feature is $K_{1}$, and the feature may be weighted as $K_{1} F_{1}$. The traditional TFIDF can effectively rank its importance according to the frequency of each feature in the tacit knowledge, but cannot consider the relationship between different feature quantities. According to the Euclidean distance algorithm, the distance value between different feature quantities is calculated, and the distance value is taken as the weight to the calculation of the feature quantity, and then the feature value of a tacit knowledge can be extracted more accurately.

\section{Pattern Recognition Tacit Knowledge Sharing System Construction}

After extracting the features of tacit knowledge, we need to build a tacit knowledge system based on this, as shown in Figure 1. When new tacit knowledge enters, if there is already a hidden knowledge feature, it is added as a supplementary content to the enterprise knowledge classification. If there is no such tacit knowledge feature in the database, it needs to be added as new knowledge. In the database, the database is updated.

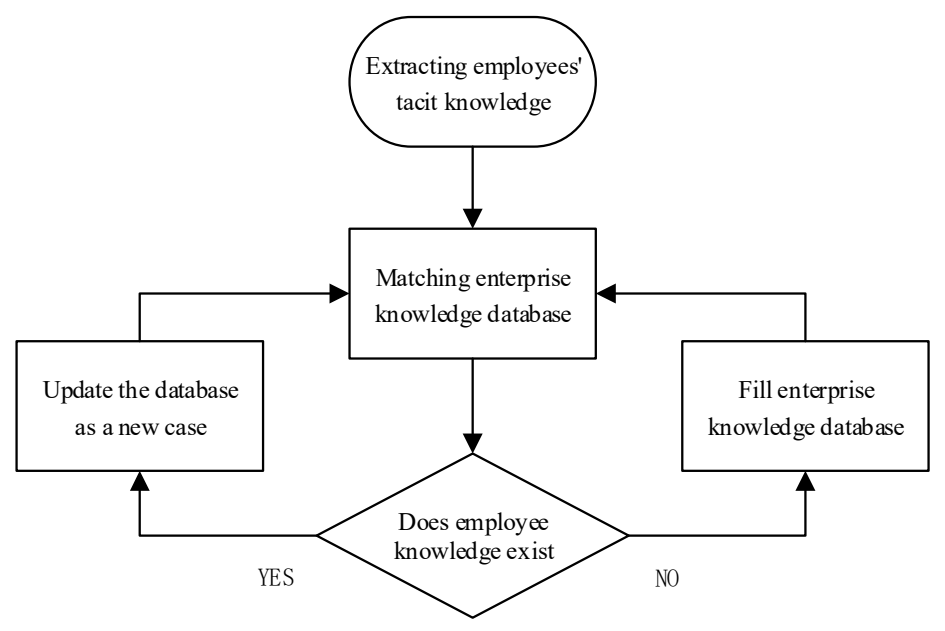

FIGURE 1. Construction flow chart of tacit knowledge sharing system

The "matching enterprise database" needs to compare the new tacit knowledge with the original content in the database. Here, the pattern recognition method is used to compare the content. Pattern recognition [13] [14] methods mainly include template matching method, ANN method, knowledge-based method, and data-based method. Here, the closest template matching method is used to perform template matching. By calculating the correlation coefficient of the template to be matched and the template to be matched, the one with the largest correlation coefficient is the optimal matching result. 


\section{CONCLUSION}

This paper proposes a TFIDF tacit knowledge feature extraction method based on Euclidean distance, aiming at the low efficiency of corporate $R \& D$ team tacit knowledge sharing under the contemporary network environment. It uses the TFIDF method to extract the characteristics of tacit knowledge and supplements it with the Euclidean distance method. Relationship weights between different features. After extracting the features of tacit knowledge, these features can be used to build a database of corporate tacit knowledge features, which can be used to continuously supplement new tacit knowledge. Therefore, a pattern recognition method is adopted to compare the new knowledge with the original knowledge. To maximize the rational use of tacit knowledge.

\section{REFERENCES}

1. Asper M. Explicit Knowledge[M]// A Companion to Greek Literature. John Wiley \& Sons, Inc, 2015:401-414.

2. Collins H M. Tacit and Explicit Knowledge[M]// Tacit and explicit knowledge /. University of Chicago Press, 2013:275-279.

3. Smith E A. The role of tacit and explicit knowledge in the workplace[J]. Journal of Knowledge Management, 2001, 5(4):311-321.

4. Newitz A. Web 3.0[J]. New Scientist, 2008, 197(2647):42-43.

5. Neches R. Enabling Technology for Knowledge Sharing[J]. Ai Magazing, 1991, 12(3):36-56.

6. Gruber T R. Toward principles for the design of ontologies used for knowledge sharing[M]. Academic Press, Inc. 1995.

7. Neches R. Enabling Technology for Knowledge Sharing[J]. Ai Magazing, 1991, 12(3):36-56.

8. Mackness J, Sui F J M, Williams R. The Ideals and Reality of Participating in a MOOC[C]// Networked Learning Conference. 2010.

9. Whalen P J, Rauch S L, Etcoff N L, et al. Masked presentations of emotional facial expressions modulate amygdala activity without explicit knowledge[J]. Journal of Neuroscience, 1998, 18(1):411-418.

10. Guyon I M, Gunn S R, Nikravesh M, et al. Feature Extraction, Foundations and Applications. Springer Verlag[J]. Studies in Fuzziness \& Soft Computing, 2006, 205(12):68-84.

11. Øivind Due Trier, Jain A K, Taxt T. Feature extraction methods for character recognition-A survey[J]. Pattern Recognition, 1995, 29(4):641-662.

12. Jing L P, Huang H K, Shi H B. Improved feature selection approach TFIDF in text mining[C]// International Conference on Machine Learning and Cybernetics, 2002. Proceedings. IEEE, 2003:944-946 vol.2.

13. Bishop C M. Neural Networks for Pattern Recognition[J]. Agricultural Engineering International the Cigr Journal of Scientific Research \& Development Manuscript Pm, 1995, 12(5):1235 - 1242.

14. Bezdek J C. Pattern Recognition with Fuzzy Objective Function Algorithms[M]. Plenum Press, 1981. 